\title{
3D-printed magnetic tweezers for dorsal traction force measurement
}

$\overline{\text { Matthew Pittman' \& Yun Chen*,1,2,3 }}$

\section{ABSTRACT}

In this study, an economic magnetic tweezers (EMT) with a sharp gradient field were designed and built, in order to facilitate accurate force measurement. Our design costs less than 40 USD and is easy to mount onto most of the microscope stages. We leverage computational fluidic dynamics techniques to calculate the forces based on the results obtained using our simple device. The EMT device is especially suitable to measure the traction forces at the dorsal side of a cell. As a proof of concept it was demonstrated that the EMT device could be applied to measure the dorsal traction forces exerted via the CD80-CTLA4 bond in metastatic cancer cells.

\section{METHOD SUMMARY}

3D-printed magnetic tweezers were developed to measure the mechanical forces transmitted at the dorsal side of a cell. We present a low-cost method to make magnetic tweezers and construct the necessary computational framework derived from the computational fluid dynamics studies to calculate the forces robustly and accurately. By tracking the displacement of magnetic particles in the microscopic field over time, dynamic forces generated by the cells can be obtained.

\section{KEYWORDS:}

3D printing $\cdot$ biomedical engineering - CTLA4 $\cdot$ dorsal traction force $\cdot$ drag force $\cdot$ force measurement $\cdot$ magnetic tweezers

'Department of Mechanical Engineering, Johns Hopkins University, MD, USA; ${ }^{2}$ Center for Cell Dynamics, Johns Hopkins University, MD, USA; ${ }^{3}$ Institute for NanoBio Technology, Johns Hopkins University, MD, USA; *Author for correspondence: yun.chen@jhu.edu
A vast body of studies in the past several decades have revealed that mechanical forces play an integral role in homeostasis and cellular function. Cells not only receive and respond to mechanical cues through a variety of mechanosignaling pathways, but also generate forces to communicate with other cells and interact with the tissue microenvironment. Forces generated by cells and external mechanical stimuli mediate many important biological processes, including cellular differentiation and proliferation, embryonic development and tissue morphogenesis, and metastasis and tumor microenvironment remodeling, to name a few. In particular, traction forces generated by cells play key roles in wound healing, angiogenesis and metastasis [1].

While there are a host of well-established methods to measure traction forces at the ventral side of the cell [2,3], including traction force microscopy [4], micro- and nano-pillar assays, and tension gauge tether assays, few methods exist to measure dorsal traction forces. However, dorsal traction forces are of biological importance too, involved in disrupting cancer cell-endothelium association [5] before intrastation, or in cell motility of neural crest during embryonic development [6], among others. In fact, laser traps method is the only technique available so far $[7,8]$. Yet, to configure a laser trap costs tens of thousands dollars at least. Magnetic tweezers, which are commonly used to probe and quantify the inherent mechanical properties of molecules or cells, can potentially be repurposed to measure dorsal traction forces. Paramagnetic particles, bound specifically to a surface molecule species at the dorsal side of the cell, experience pulling forces due to the gradient field emanating from the magnetic tweezers; the displacement and velocity of these particles can then be analyzed to determine the dorsal traction force. However, most magnetic tweezer configurations include electromagnetic coils, power supplies and/or stepper motors [9-12]. For many research groups, such configurations are prohibitive in cost, and they often require long periods of guided training for successful operation. In this study, we detail the design and construction of affordable and easy-touse magnetic tweezers, coined economic magnetic tweezers (EMT), and demonstrate that they can be used to measure the traction forces at the dorsal side of the cell.

The EMT, which cost less than $\$ 40$ in materials and can be readily mounted to any microscope stage, consist of four parts: a base, a magnet mount, a magnet and a needle. The needle runs across the magnet, and the tip, which acts as the magnetic field-generating probe, rests on the sample. The magnet and needle are secured in the magnet mount, which has one rotational degree of freedom around the base so that the probe can be raised and lowered onto the sample (Figure 1A and Supplementary Data). The base, which is simply taped to the stage, can be made in different shapes and sizes so that the device can be mounted on any type of microscope stage.

The EMT were designed to be quickly, easily and affordably built. The magnet and needle that fit our specifications are available online, and the base and magnet mount can be 3D printed. Specifically, we used an iron tattoo needle (\#9 Round Shader), which is highly magnetizable and has a sharp tip of $\sim 50 \mu \mathrm{m}$ diameter, resulting in a sharp field gradient for force generation. The neodymium magnet (K\&J Magnetics, DX48-N52) is cylindrical with a surface field strength of $0.4 \mathrm{~T}$. Magnets of different strengths can be placed into the holder. The needle position can be adjusted along the magnet. This not only enables the device to fit on a greater range of stages, but also offers the user control over the strength of the magnetic field at the probe tip. The base and magnet mount were printed in ABS plastic by Stratasys uPrint. 
(A)

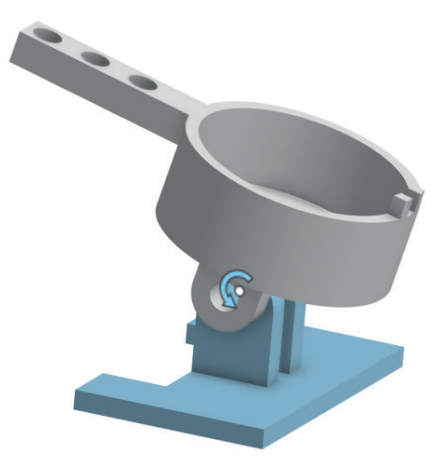

(B)

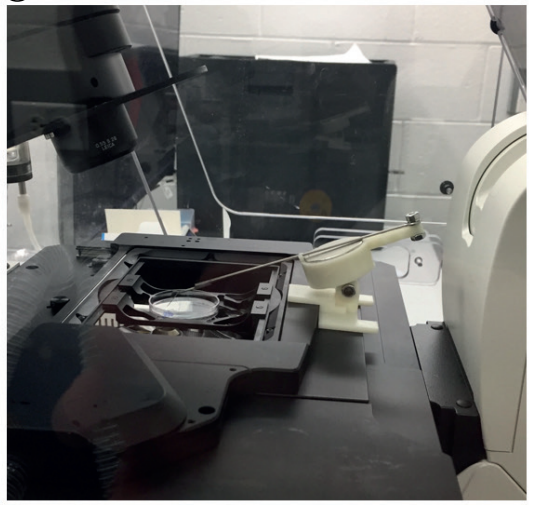

Figure 1. Design and application of the economic magnetic tweezers. (A) The magnetic tweezers housing consists of two parts: a supporting base and a mount to accommodate the neodymium magnet and iron needle. Both parts are 3D printed. The magnet/needle housing can be rotated around the base so that the pole tip returns to the center of the objective after changing the sample. (B) The economic magnetic tweezers are mounted on a microscope stage for live cell imaging.

Once assembled, the EMT can be mounted by taping its base to the microscope stage. Using an empty slide or dish in place of the sample, the probe tip is rotated down into the position above the objective, and the stage can be adjusted in $\mathrm{x}, \mathrm{y}$ and $\mathrm{z}$ to ensure that the tip is in focus and is centered in the field of view (Figure 1B). With the EMT aligned, the empty dish is replaced with the sample for imaging.

As a proof-of-concept, EMT were used to study the traction forces transmitted through the CTLA4-CD80 bond at the dorsal side of metastatic MDA-MB-231 breast cancer cells. CTLA4 is known to be expressed at the cell surface and binds to CD80 at high affinity [13]. It has been observed that CD80, usually expressed on the surface of another cell, will undergo transendocytosis [14], which is powered by the traction force generated at the dorsal side of the cell. Traction force can be transmitted by catch bond [15], where the surface receptor linked to the actomyosin cytoskeleton is required to be mechanically engaged. It is assumed that the CTLA4-CD80 bond utilizes a catch bond mechanism, and this bond can be mechanically activated by engaging CTLA4 with CD80-coated paramagnetic particles subjected to the field gradient of the EMT.

To fabricate CD80-coated paramagnetic particles, we conjugated recombinant CD80-Fc (Biolegend 555404) onto 2.8- $\mu \mathrm{m}$ Dynabeads using Protein $\mathrm{G}$ (ThermoFischer 10003D). CD80-Fc and the Dynabeads were mixed at a ratio of 5:1 CD80-Fc molecules to total Protein $\mathrm{G}$ available. Following appropriate washing and purification, approximately $10^{4}$ particles were added to the MDA-MB-231 cells cultured on $30-\mathrm{mm}$ glass bottom dishes at $70-80 \%$ confluence. The sample was incubated for 2 min to allow bond formation between CD80 and CTLA4. Timelapse images were then acquired using a Leica SP8 microscope recording at a sampling rate of 20 frames/sec.

To analyze the dorsal traction forces generated by cells, the EMT were first calibrated for the experimental parameters by calculating the drag force experienced by freely moving magnetic particles. To minimize the errors introduced by the motion in the z-axis, we only recorded the movement of particles in the same focal plane as that of the needle tip. The drag force on a spherical particle was calculated using Stokes' Law: $F_{\text {drag }}=3 \pi \eta d V$, where $\eta$ represents dynamic viscosity, $d$ particle diameter, and $V$ particle velocity. The velocity of the particles as they travel toward the probe tip was determined with either manual or automatic tracking (Figure 2A) using ImageJ. The drag force calculated based on the free particle velocity was then plotted as a function of distance from the tip to determine the quantitative relationship between distance from the tip and force generated by the EMT. The EMT calibration curve was determined by fitting the data to a single-phase exponential decay model. The result showed that in the presence of EMT, the Dynabeads experience the force-distance relation $F \sim F_{\max } e^{-K x}$, $\left(\mathrm{R}^{2}=.9849\right)$, where $F$ represents the force exerted on the particle, $x$ the distance of the particle from the probe tip, and $F_{\text {max }}$ and $K$ are fit coefficients (Figure 2B).

Once the EMT have been calibrated, they can be used to study contractile forces generated by cells. For a cell pulling the paramagnetic particle away from the tip, the force generated by the cell can be calculated as:

(Eq. 1)

$$
F_{\text {cell }}=F_{E M T}+F_{\text {drag }}
$$

so, the dorsal traction force generated by the cell is the sum of the magnetic force on the particles, determined from the calibration curve as a function of the distance of the cell from the pole tip, and the drag force on the moving particle (Figure $2 \mathrm{C}$ ).

Since the particles are positioned at the interface between the cell membrane and medium, the viscosity of the cell membrane must be factored in when calculating the drag force. Depending on the portion of the particle embedded in cell, which can be determined by angle $\theta$ (Figure 2E), $F_{\text {drag }}$ can be evaluated as:

(Eq. 2)

$$
F_{\text {drag }}=\frac{1}{2} \cdot\left(1+\frac{9}{16} \cdot \cos \theta-0.139 \cdot \cos ^{2} \theta\right) \cdot 3 \pi \eta d V
$$

where the first three terms account for the correction coefficient for particles moving at the interface [16]. A representative experiment is shown in Figure 2C, where the Dynabead moves in the opposite direction of the magnetic field gradient at $0.3 \mu \mathrm{m} / \mathrm{s}$. After 3D image reconstruction, it is determined that the particle is embedded in the cell membrane at the contact $\theta=\pi / 3$ (Figure 2D) and that the membrane's viscosity is $80 \mathrm{~Pa} \cdot \mathrm{S}$ [17]. $F_{\text {cell }}$ was estimated to be $435 \mathrm{pN}$. The result is comparable to a previous optical traps study, where $158 \mathrm{pN}$ of pulling force generated by actomyosin cytoskeleton, although transmitted through a different ligand-receptor bond (integrin-fibronectin), was detected on 1- $\mu \mathrm{m}$ particles [7]. The accuracy of our method depends on the accuracy of $\theta$ value. Usually $\theta$ ranges from $\pi / 3$ to $2 \pi / 3$; by assuming an inaccurate $\theta$ value, the error can be as big as $33 \%$. Therefore, measurement of $\theta$ 


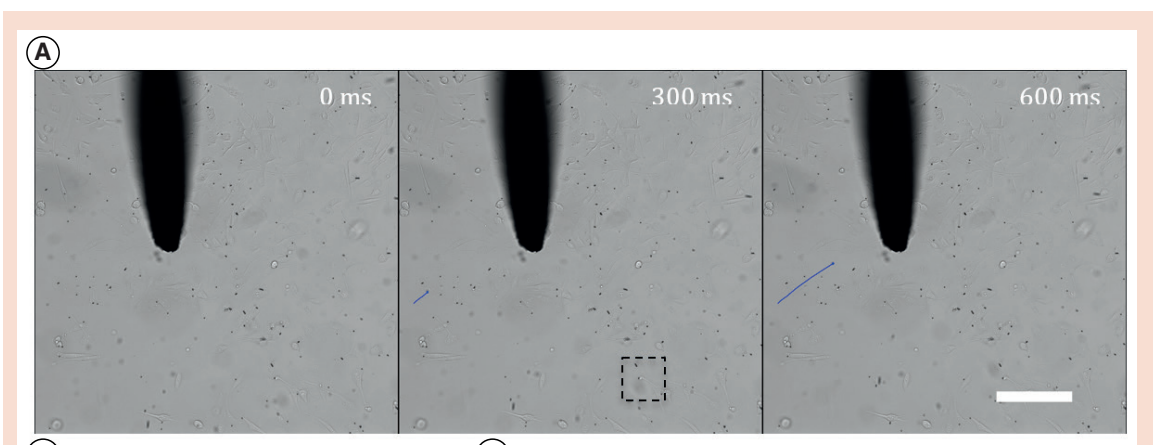

(B)

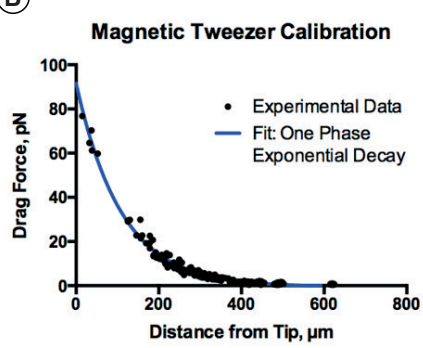

(D)

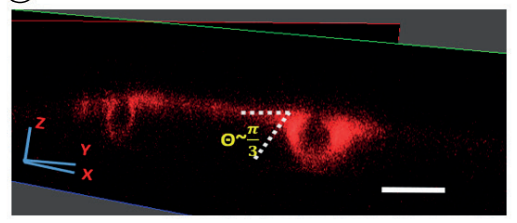

(c)

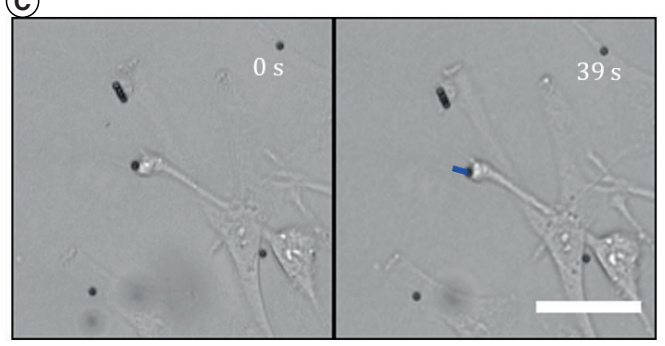

(E)

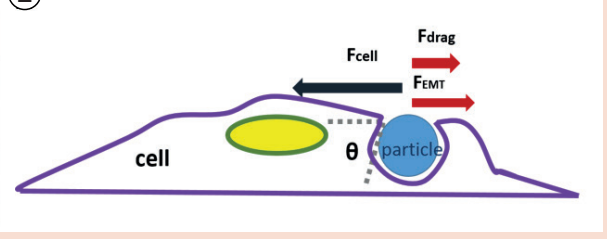

Figure 2. Force field calibration and cellular force measurement. (A) Timelapse images of paramagnetic particles moving in the direction of the probe tip. Particle velocity is used for force field calibration. Scale: $200 \mu \mathrm{m}$. (B) Force exerted on the particles is plotted as a function of distance from the probe tip. The data points are fitted with a one-phase exponential decay model $\left(R^{2}=.9849\right)$. (C) The magnified area (dashed box in A) reveals that the cell continuously pulls the particle in the opposite direction of the magnetic field gradient imposed by the magnetic tweezers. This force can be quantified as $\mathrm{F}_{\text {cell }}=\mathrm{F}_{\mathrm{EMT}}+\mathrm{F}_{\text {drag }}$; in this case, $\mathrm{F}_{\text {cell }} \approx 254.7 \mathrm{pN}$. Scale: $50 \mu \mathrm{m}$. (D) A $3 \mathrm{D}$-reconstruction image shows that the particle is enveloped by the cell membrane, forming a $60^{\circ}$ contact angle between the particle, the cell and medium. Scale bar: $5 \mu \mathrm{m},(\mathrm{E})$ The schematic shows the particle moving at the cell-medium interface.

- by $3 \mathrm{D}$ imaging is recommended prior to calculation.

In summary, we present a robust platform by integrating computational dynamic fluid techniques and the EMT for dorsal traction force measurement at considerably less cost. In addition, the EMT can also be used to examine a variety of other forces on the dorsal side of the cell. For example, rupture forces for the ligand-receptor binding can be determined by quantifying the force required to detach the particles from cells. Due to its low cost, modular design and easeof-construction, we envision that EMT will find widespread applications in the cell biology community.

\section{SUPPLEMENTARY DATA}

To view the supplementary data that accompany this paper please visit the journal website at: www.future-science. com/doi/suppl/10.2144/btn-2018-0078. The file contains the design used for the printable parts of the EMT in .stl format.

\section{AUTHOR CONTRIBUTIONS}

MP and YC formulated the concepts and designed the experiments. MP and YC performed the experiments and data analysis. MP and YC wrote the manuscript.

\section{FINANCIAL \& COMPETING INTERESTS DISCLOSURE}

The authors have no relevant affiliations or financial involvement with any organization or entity with a financial interest in or financial conflict with the subject matter or materials discussed in the manuscript. This includes employment, consultancies, honoraria, stock ownership or options, expert testimony, grants or patents received or pending, or royalties.

No writing assistance was utilized in the production of this manuscript.

\section{OPEN ACCESS}

This work is licensed under the AttributionNonCommercial-NoDerivatives 4.0 Unported License. To view a copy of this license, visit http://creativecommons.org/licenses/ by-nc-nd/4.0/

\section{REFERENCES}

1. Wang $\mathrm{JH}-\mathrm{C}$, Lin J-S. Cell traction force and measurement methods. Biomech. Model. Mechanobiol. 6(6), 361-371 (2007).

2. Polacheck WJ, Chen CS. Measuring cell-generated forces: a guide to the available tools. Nat. Methods 13(5), 415-423 (2016)

3. Wang $X$, Sun J, Xu Q et al. Integrin molecular tension within motile focal adhesions. Biophys. J. 109(11) 2259-2267 (2015).

4. Colin-York H, Eggeling C, Fritzsche M. Dissection of mechanical force in living cells by super-resolved traction force microscopy. Nat. Protoc. 12(4), 783-796 (2017).

5. Reymond N, d'Água BB, Ridley AJ. Crossing the endothelial barrier during metastasis. Nat. Rev. Cancer 13(12), 858-870 (2013)

6. Meloty-Kapella L, Shergill B, Kuon J, Botvinick E, Weinmaster $\mathrm{G}$. Notch ligand endocytosis generates mechanical pulling force dependent on dynamin, epsins, and actin. Dev. Cell. 22(6), 1299-1312 (2012).

7. Galbraith CG, Sheetz MP. Keratocytes pull with similar forces on their dorsal and ventral surfaces. J. Cell Biol. 147(6), 1313-1324 (1999).

8. Choquet D, Felsenfeld DP, Sheetz MP. Extracellular matrix rigidity causes strengthening of integrin-cytoskeleton linkages. Cell 88(1), 39-48 (1997).

9. Lipfert J, Lee M, Ordu O, Kerssemakers JWJ, Dekker NH. Magnetic tweezers for the measurement of twist and torque. J. Vis. Exp. 19(87), 1-18 (2014).

10. De Vlaminck I, Henighan T, van Loenhout MTJ et al. Highly parallel magnetic tweezers by targeted DNA tethering. Nano Lett. 11(12), 5489-5493 (2011)

11. Tanase $M$, Biais $N$, Sheetz $M$. Magnetic tweezers in cell biology. Methods Cell Biol. 83, 473-493 (2007).

12. Neuman KC, Nagy A. Single-molecule force spectroscopy. optical tweezers, magnetic tweezers and atomic
force microscopy. Nat. Methods. 5(6), 491-505 (2008).

13. Contardi E, Palmisano GL, Tazzari PL et al. CTLA-4 is constitutively expressed on tumor cells and can trigger apoptosis upon ligand interaction. Int. J. Cancer 117(4), 538-550 (2005).

14. Qureshi OS, Zheng Y, Nakamura K et al. Trans-endocytosis of CD80 and CD86: a molecular basis for the cell-extrinsic function of CTLA-4. Science 332(6029), 600-603 (2011).

15. Bangasser BL, Rosenfeld SS, Odde DJ. Determinants of maximal force transmission in a motor-clutch model of cell traction in a compliant microenvironment. Biophys. J. 105(3), 581-592 (2013)

16. Dörr A, Hardt S, Masoud H, Stone HA. Drag and diffusion coefficients of a spherical particle attached to a fluidfluid interface. J. Fluid Mech. 790, 607-618 (2016).

17. Simunovic $M$, Manneville J-B, Renard $H-F$ et al. Friction mediates scission of tubular membranes scaffolded by BAR proteins. Cell 170(1), 172-184.e11 (2017).

First draft submitted: 1 June 2018; Accepted for publication: 17 September 2018 